\title{
Die innere Uhr der Haut
}

Forscher der Berliner Charité und eines Hamburger Unternehmens haben entdeckt, dass die menschliche Haut einen Taktgeber besitzt, der die zeitliche Steuerung von Regenerations- und Reparaturprozessen koordiniert.

D ie Arbeitsgruppen um Achim Kramer (Chronobiologie, Charité Berlin) und Thomas Blatt (Hamburger Hautforschungszentrum) untersuchten, wie sich die Haut den tageszeitabhängigen Bedingungen anpasst. Dazu entnahmen sie jungen, gesunden Probanden zu verschiedenen Tageszeitpunkten Keratinozyten.

Die Analyse des Gesamtgenoms dieser Zellen mittels eines speziellen Micro-Arrays ergab, dass mehrere hundert Transkriptionsfaktoren für die Regeneration und Reparatur der Haut einem zirkadianen Rhythmus unterliegen.

Einer dieser Faktoren mit der Bezeichnung „Krüppel-like-factor 9“ (Klf9) verzögert die Mitose der Keratinozyten. Bei experimenteller Reduktion dieses Faktors in der Zellkultur war eine schnellere Keratinozyten-Proliferation nachweisbar. Analog zeigte sich eine verringerte Zellteilungsrate bei verstärkter Aktivität von

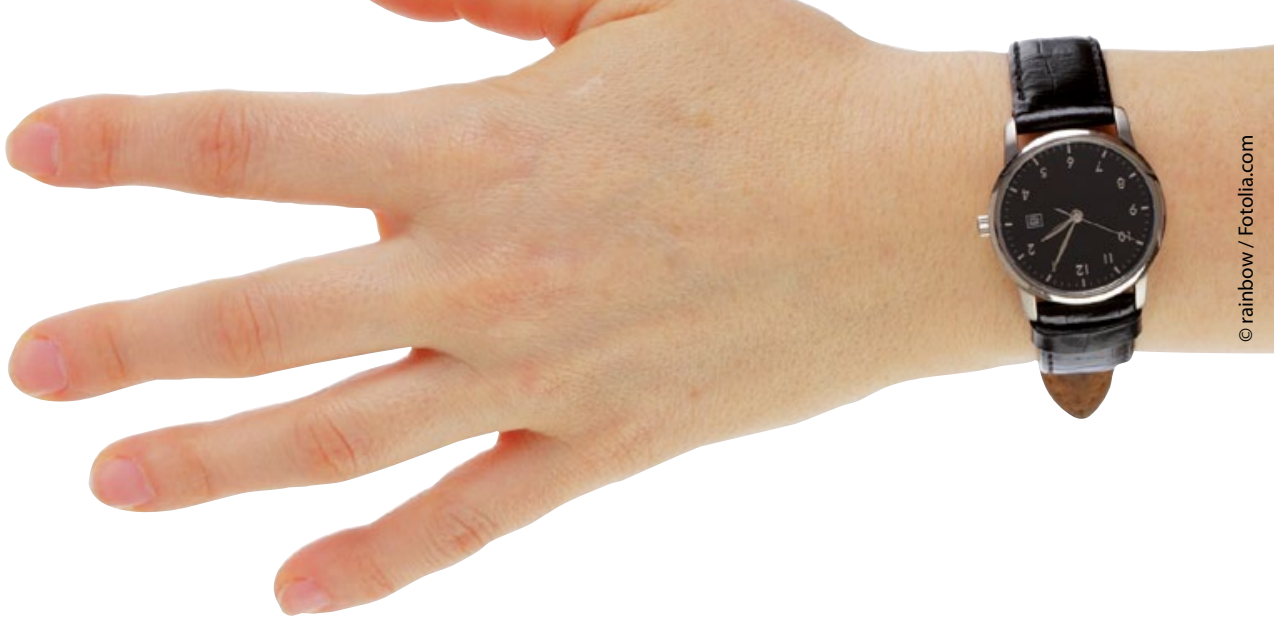

Klf9. Dieser Transkriptionsfaktor wird in seiner Aktivität vor allem durch Kortisol beeinflusst.

Die mutmaßlichen Zielgene von Klf9 sind unter anderem für die Expression von Proliferations- und Differenzierungsmarkern verantwortlich, die auch in vivo einem zirkadianen Rthythmus folgen.

Kramer sieht in den Ergebnissen der Publikation eine Vielzahl von zukünftigen Anwendungsmöglichkeiten. Bei einem noch genaueren Verständnis der Prozesse wäre es beispielsweise denkbar, „Medikamente gezielt zu den Tageszeiten einzusetzen, an denen sie am besten wirken und die wenigsten Nebenwirkungen haben."
Fazit: Der Sinn einer biologischen Uhr besteht darin, ein exaktes Timing unterschiedlicher Prozesse wie Proliferation, Differenzierung und DNA-Reparatur von Zellen zu steuern und aufeinander abzustimmen. Gerade für die Haut, die vielen, auch tageszeitabhängigen Umwelteinflüssen unterworfen ist, ist dies möglicherweise besonders wichtig. Welche klinischen Anwendungsmöglichkeiten die zirkadiane Rhythmik von Transkriptionsfaktoren und deren Zielgene ergeben, muss sich noch zeigen. Dr. Andreas Fischer

Spörl F et al. Krüppel-like factor 9 is a circadian transcription factor in human epidermis that controls proliferation of keratinocytes. Proc Natl Acad Sci USA 2012; 109: 10903-8

\section{Digitaler Blick auf melanozytäre Hautläsionen}

Die digitale Dermatoskopie bietet bei Verdacht auf ein Melanom auch in der täglichen Praxis Vorteile: Im Vergleich zur alleinigen Anwendung der optischen Dermatoskopie lassen sich unnötige Exzisionen reduzieren und maligne Hautveränderungen früher entdecken.

$\mathrm{B}$ elgische Dermatologen haben den zusätzlichen Nutzen der digitalen Dermatoskopie über einen Zeitraum von einem Jahr untersucht. 46 Ärzte nahmen an der Studie teil und wurden drei Gruppen zugeordnet. In zwei Gruppen, die ohne digitale Dermatoskopie arbeiteten, waren 24 Ärzte mit geringer (A) und zwölf Ärzte mit großer Erfahrung (B) in der Anwendung der optischen Dermatoskopie. Die dritte Gruppe (C) bestand aus Ärzten, die viel Erfahrung besaßen und zusätzlich die digitale Dermatoskopie nutzten. In die Auswertung flossen die Befunde bei insgesamt 1.851 Läsionen, einschließlich 231 Melanomen, von 1.713 Patienten ein.

Primärer Endpunkt der Untersuchung war das Melanom/Nicht-Melanom-Verhältnis (M/NM-Ratio) unter den exzidierten Läsionen. In den drei Gruppen lag dieser Wert bei $1: 10,74$ (Gruppe A), $1: 8,14$ (Gruppe B) und $1: 2,43$ (Gruppe C). Damit schnitten die Ärzte der Gruppe $\mathrm{C}$ signifikant besser als ihre Kollegen aus den anderen beiden Gruppen $a b$.

Dermatologen, die die digitale Dermatoskopie nutzten, entdeckten invasive Melanome im Mittel bereits bei einer signifikant geringeren Eindringtiefe (Breslow-
Index) als Kollegen, die sich nur auf die optische Dermatoskopie verlassen konnten - 0,32 mm versus 0,90 mm. Die Auswertung der Daten ergab schließlich, dass sich die Zahl unnötiger Exzisionen durch die zusätzliche Anwendung der digitalen Dermatoskopie um mehr als das Dreifache reduzieren lässt.

Fazit: Bei Nutzung der digitalen Dermatoskopie konnten Kollegen signifikant besser Melanome gegenüber Nicht-Melanomen differenzieren als nur durch optische Dermatoskopie. Allerdings ersetzt die digitale Technik auch hier nicht die klinische Erfahrung. Peter Leiner

Tromme I. et al. Availability of digital dermoscopy in daily practice dramatically reduces the number of excised melanocytic lesions: results from an observational study. $\mathbf{B r} \mathbf{J}$ Dermatol 2012 May 7; Epub ahead of print 